\title{
Determination of Useful Ranges of Mixing Conditions for Glycerin Fatty Acid Ester by Multiple Regression Analysis
}

\author{
Takeaki Uchimoto, Yasunori Iwao, Hiroaki Hattori, Shuji Noguchi, and Shigeru Itai* \\ Department of Pharmaceutical Engineering, School of Pharmaceutical Sciences, University of Shizuoka; 2-1 Yada, \\ Suruga-ku, Shizuoka 422-8526, Japan. \\ Received June 13, 2013; accepted September 3, 2013
}

\begin{abstract}
The interaction of the effects of the triglycerin full behenate (TR-FB) concentration and the mixing time on lubrication and tablet properties were analyzed under a two-factor central composite design, and compared with those of magnesium stearate $(\mathrm{Mg}-\mathrm{St})$. Various amounts of lubricant $(0.07-3.0 \%)$ were added to granules and mixed for $1-30$ min. A multiple linear regression analysis was performed to identify the effect of the mixing conditions on each physicochemical property. The mixing conditions did not significantly affect the lubrication properties of TR-FB. For tablet properties, tensile strength decreased and disintegration time increased when the lubricant concentration and the mixing time were increased for Mg-St. The direct interaction of the Mg-St concentration and the mixing time had a significant negative effect on the disintegration time. In contrast, any mixing conditions of TR-FB did not affect the tablet properties. In addition, the range of mixing conditions which satisfied the lubrication and tablet property criteria was broader for TR-FB than that for Mg-St, suggesting that TR-FB allows tablets with high quality attributes to be produced consistently. Therefore, TR-FB is a potential lubricant alternative to $\mathrm{Mg}-\mathrm{St}$.
\end{abstract}

Key words glycerin fatty acid ester; magnesium stearate; experimental design; multiple regression analysis; process optimization

Magnesium stearate $(\mathrm{Mg}-\mathrm{St})$ is a widely used lubricant in tablet manufacturing; however, the concentration and mixing time affect tablet quality attributes, including hardness, ${ }^{1)}$ disintegration time, ${ }^{2-4)}$ and drug dissolution. ${ }^{5,6)}$ In addition, variation from batch to batch and between brands associated with moisture content ${ }^{7,8)}$ and specific surface area ${ }^{9)}$ also influence the lubrication properties and the consequent tablet quality attributes, which means it is difficult to control the mixing conditions. $^{10)}$

A variety of substances, including hydrophilic organic materials such as sodium stearyl fumarate, ${ }^{11,12}$ magnesium lauryl sulfate, ${ }^{13)}$ and inorganic materials such as hexagonal boron nitride, ${ }^{4}$ have been evaluated as alternative lubricants to $\mathrm{Mg}$-St. In general, mixing conditions, such as the lubricant concentration and the mixing time, are crucial factors affecting the lubrication and tablet properties. However, the detailed interaction between the concentration and the mixing time on the lubrication and tablet properties has not been investigated. It is required to examine the useful range of mixing conditions of the lubricant and its effect on the lubrication and tablet properties.

Recently, we have reported two types of glycerin fatty acid ester lubricants, triglycerin full behenate (TR-FB) and triglycerin half behenate (TR-HB), as potential alternatives to $\mathrm{Mg}-\mathrm{St}^{14-16)}$ Generally, Mg-St is used at a concentration of $0.5 \%$ during the manufacturing process, but it is necessary to discard initial tablets after starting compression until the uniform lubrication effect takes place. In our previous study, it was demonstrated that the lubrication performance of TR-FB and TR-HB at the same concentration was better immediately after the start of compression than that of $\mathrm{Mg}-\mathrm{St} .{ }^{15)}$ In addition, decreased tablet tensile strength (TS), prolonged disintegration time (DT), or retarded drug dissolution was not observed for TR-FB and TR-HB at a mixing time of $5 \mathrm{~min}$, even when the

The authors declare no conflict of interest. lubricant concentration was increased to $3.0 \%$. In contrast, these properties were deteriorated for $\mathrm{Mg}-\mathrm{St} .{ }^{15,16)}$ Therefore, the use of TR-FB or TR-HB as a lubricant produced tablets with uniform quality and without the pharmaceutical problems associated with Mg-St. However, only limited mixing conditions of TR-FB and TR-HB were examined similar to previous lubrication studies. In addition, according to the Food and Drug Administration's Pharmaceutical Good Manufacturing Practice for the 21st Century initiative, critical process parameters influencing quality attributes must be characterized and quality assurance in manufacturing processes are hoped to be controlled. ${ }^{17)}$ Therefore, for TR-FB, the relationship between the mixing conditions and the tablet properties and the useful range of mixing conditions must be comprehensively clarified in order to evaluate TR-FB as a novel lubricant.

A combination of experimental design and multiple regression analysis is required to determine the interactions between the operational conditions and to optimize the process conditions. Previously, we have successfully clarified the effects of the process parameters and their interactions on the manufacture of solid oral dosage forms, such as functional fine granules and orally disintegrating tablets using the combination. $^{18-20)}$ Thus, experimental design and multiple regression analysis should also be suitable for evaluating the lubrication properties of TR-FB. Therefore, in this study, we changed lubricant mixing at various concentrations and mixing times, according to a two-factor central composite design, and then prepared tablets. A multiple linear regression analysis was conducted to verify the relationship between the mixing conditions and the lubrication properties, such as the pressure transmission ratio (PTR) and ejection force (EF), and the tablet properties, such as TS and DT. The useful range of mixing conditions that satisfied both the lubrication and tablet properties was also determined in order to quantitatively evaluate the potential of TR-FB as an alternative to $\mathrm{Mg}$-St. 


\section{Experimental}

Materials Mg-St (vegetable origin, specifications as a drug additive, listed in the Japanese Pharmacopoeia Sixteenth Edition (JP16)) was purchased from Wako Pure Chemical Industries, Ltd. (Osaka, Japan). TR-FB (Poem TR-FB ${ }^{\circledR}$, vegetable origin, specifications as a food additive) was kindly provided by Riken Vitamin Co., Ltd. (Tokyo, Japan). The chemical structure and other physicochemical properties, such as particle size, specific surface area, melting point and moisture content of lubricants, have previously been reported. ${ }^{14,15)}$ Lactose monohydrate (DFE Pharma Co., Ltd., Tokyo, Japan) and corn starch (Nihon Shokuhin Kakou Co., Ltd., Tokyo, Japan) were used as fillers, and solid-state hydroxypropylcellulose (HPC-L ${ }^{\circledR}$, Nippon Soda Co., Ltd., Tokyo, Japan) was used as a binder. All other excipients used in this study are listed in JP16.

Granulation Lactose monohydrate and corn starch were dried for $12 \mathrm{~h}$ at $50^{\circ} \mathrm{C}$ in an oven. Lactose monohydrate $(350 \mathrm{~g})$ and corn starch $(150 \mathrm{~g})$ were mixed for $10 \mathrm{~min}$ using a mixer (Fuji Medical Equipment Co., Ltd.). The ratio of lactose monohydrate and corn starch was the standard formulation given by the Society of Powder Technology, Japan, Division of Particulate Design and Preparations. Aqueous 5.0\% HPC-L $(100 \mathrm{~g})$ was sprayed onto the powder, and the mixture was kneaded for $10 \mathrm{~min}$. Granulation was performed using a rotary squeeze-type granulator with a sieve size of $0.8 \mathrm{~mm}$ (Hata Iron Works Co., Ltd., Kyoto, Japan). The granules were dried in an oven at $50^{\circ} \mathrm{C}$ for $12 \mathrm{~h}$ or longer, and then sieved through a $1680 \mu \mathrm{m}$ sieve. The granules that did not pass through a $350 \mu \mathrm{m}$ sieve were collected. This process was repeated several times, and the granules were then mixed uniformly and subjected to the experiments.

Experimental Design, Tablet Preparation and Determination of Pressure Transmission Ratio and Ejection Force The granules $(100 \mathrm{~g})$ were fed into a V-shaped mixer (S3 stainless steel mixer, Tsutsui Scientific Instruments Co., Ltd., Tokyo, Japan) and their surface was flattened. Mg-St or TR-FB was added to the center of the granules and mixed at a rotation rate of $35 \mathrm{rpm}$. A two-factor central composite design was used to analyze the relationship between the lubricant mixing conditions and their lubrication and tablet properties.
The lubricant concentration $\left(X_{1}\right)$ and the lubricant mixing time $\left(X_{2}\right)$ were used as independent variables. In preliminary work, when Mg-St or TR-FB was mixed with the granules at concentrations of 3.0, 4.0, 5.0, and 6.0\%, PTR plateaued, and it was also found that a tablet was obtained even at a concentration of $0.07 \%$ for each lubricant (unpublished results). Furthermore, PTR and EF were unchanged for mixing times between 30 and $60 \mathrm{~min}^{15}$ ) Therefore, lubricant concentrations of $0.07-3.0 \%$ and mixing times of $1-30 \mathrm{~min}$ were used in the present study. The normalized factor levels of the independent variables and the conditions for each batch are listed in Table 1.

The tablets were prepared on a single punch tablet machine (N30-EX, Okada Seiko Co., Ltd., Tokyo, Japan), and had a diameter of $8 \mathrm{~mm}$ (flat-faced punch) and weighed $200 \mathrm{mg}$. The tableting speed was 10 tablets per minute and the tableting force was $10 \mathrm{kN}$. The pressure of the punch during tableting was measured by using a load cell with a data recorder (Daatsu II, Okada Seiko Co., Ltd.), and was converted to the applied force. PTR was calculated as the ratio of maximum pressure of the lower punch to maximum pressure of the upper punch. EF, which was the force applied to the lower punch during tablet ejection, was measured with a $2 \mathrm{kN}$ load cell.

Determination of Tablet Tensile Strength and Disintegration Time Ten tablets were selected at random for the TS measurements. The TS of the tablets was determined by diametrical compression tests using a hardness meter with a $300 \mathrm{~N}$ load cell (precision of 1 N, PC-30, Okada Seiko Co., Ltd.) in order to accurately measure the maximal diametrical crushing force $(F)$. The diameter and thickness of tablets were measured using a micrometer with precision of $0.01 \mathrm{~mm}$ (500-302 CD-20, Mitutoyo Corporation, Kanagawa, Japan). The TS $(\sigma)$ was calculated by the following formula ${ }^{21)}$ :

$$
\sigma=2 \cdot F /(\pi \cdot D \cdot t)
$$

where $D$ and $t$ are the diameter and the thickness of tablets, respectively.

Six tablets were selected at random from the prepared tablets for the disintegration test, and DT was determined according to the JP16 disintegration test using a disintegration tester

Table 1. Experimental Design

\begin{tabular}{|c|c|c|c|c|}
\hline \multirow{2}{*}{ Batch No. } & \multicolumn{2}{|c|}{ Lubricant concentration $\left(X_{1}\right)$} & \multicolumn{2}{|c|}{ Mixing time $\left(X_{2}\right)$} \\
\hline & $\%$ & Level & $\min$ & Level \\
\hline 1 & 0.07 & $-\sqrt{2}$ & 15.5 & 0 \\
\hline 2 & 0.50 & -1 & 5.2 & -1 \\
\hline 3 & 0.50 & -1 & 25.8 & 1 \\
\hline 4 & 1.53 & 0 & 1.0 & $-\sqrt{2}$ \\
\hline 5 & 1.53 & 0 & 15.5 & 0 \\
\hline 6 & 1.53 & 0 & 15.5 & 0 \\
\hline 7 & 1.53 & 0 & 15.5 & 0 \\
\hline 8 & 1.53 & 0 & 30.0 & $\sqrt{2}$ \\
\hline 9 & 2.57 & 1 & 5.2 & -1 \\
\hline 10 & 2.57 & 1 & 25.8 & 1 \\
\hline 11 & 3.00 & $\sqrt{2}$ & 15.5 & 0 \\
\hline
\end{tabular}


(Toyama Sangyo Co., Ltd., Osaka, Japan). Distilled water at $37.0 \pm 0.5^{\circ} \mathrm{C}$ was used as the test fluid.

Statistical Analysis The multiple linear regression analysis and optimization was performed using JMP 9 (SAS Institute Japan Ltd., Tokyo, Japan). A linear regression was performed on the data for each characteristic as a function of the two process parameters and their interactions. The response surfaces were constructed in Maple 13 (Waterloo Maple Inc., ON, Canada).

\section{Results and Discussion}

Characterization of Lubrication and Tablet Properties and Multiple Regression Analysis Table 2 shows the lubrication and tablet properties of all 11 batches. Multiple linear regression analysis was performed on these data in order to examine the significance of each operational factor and their effect on the lubrication properties and tablet properties (Tables 3, 4). The relationship between the main factors and their interactions with the results were determined, and are presented as quadratic equations of the general form:

$$
Y=a_{1} X_{1}+a_{2} X_{2}+a_{3} X_{1}^{2}+a_{4} X_{2}^{2}+a_{5} X_{1} X_{2}
$$

where $a_{1}, a_{2}, a_{3}, a_{4}$, and $a_{5}$ are the coefficients of each term. Because the coefficients were calculated using the coded values (Table 1), the various terms can be compared directly. Therefore, the coefficients represent the positive or negative effects of the two parameters and their interactions against each final property. Tables 3 and 4 contain the coefficients; the $p$-value obtained by the $t$-test to assess the significance of each term; and the $R^{2}$ value, which indicates the goodness of fit of each linear regression equation.

Table 2. Lubrication Properties and Tablet Properties

\begin{tabular}{|c|c|c|c|c|c|c|c|c|}
\hline \multirow{2}{*}{ Batch No. } & \multicolumn{4}{|c|}{$\mathrm{Mg}-\mathrm{St}$} & \multicolumn{4}{|c|}{ TR-FB } \\
\hline & PTR (\%) & $\mathrm{EF}(\mathrm{N})$ & TS (MPa) & DT (s) & PTR (\%) & $\mathrm{EF}(\mathrm{N})$ & $\mathrm{TS}(\mathrm{MPa})$ & DT (s) \\
\hline 1 & 85.7 & 96.5 & 1.80 & 150 & 89.5 & 100.3 & 1.41 & 148 \\
\hline 2 & 90.4 & 66.7 & 1.47 & 198 & 92.5 & 73.4 & 1.38 & 112 \\
\hline 3 & 90.4 & 74.6 & 1.18 & 186 & 92.5 & 65.5 & 1.55 & 138 \\
\hline 4 & 91.7 & 71.8 & 1.43 & 209 & 92.3 & 76.5 & 1.49 & 90 \\
\hline 5 & 90.6 & 70.2 & 0.76 & 266 & 92.3 & 74.2 & 1.43 & 120 \\
\hline 6 & 90.3 & 67.1 & 0.86 & 281 & 90.0 & 73.8 & 1.58 & 108 \\
\hline 7 & 90.7 & 67.3 & 0.82 & 248 & 91.2 & 70.0 & 1.56 & 119 \\
\hline 8 & 90.5 & 57.0 & 0.86 & 551 & 92.4 & 75.3 & 1.50 & 119 \\
\hline 9 & 89.9 & 60.1 & 0.83 & 352 & 90.0 & 74.3 & 1.57 & 98 \\
\hline 10 & 91.1 & 53.1 & 0.84 & 657 & 90.3 & 73.8 & 1.48 & 104 \\
\hline 11 & 91.5 & 50.6 & 1.01 & 703 & 90.2 & 80.2 & 1.55 & 89 \\
\hline
\end{tabular}

Table 3. Multiple Regression Analysis of Lubrication Properties

\begin{tabular}{|c|c|c|c|c|c|c|c|c|}
\hline \multirow{3}{*}{ Term } & \multicolumn{4}{|c|}{$\mathrm{Mg}-\mathrm{St}$} & \multicolumn{4}{|c|}{ TR-FB } \\
\hline & \multicolumn{2}{|c|}{ PTR } & \multicolumn{2}{|c|}{$\mathrm{EF}$} & \multicolumn{2}{|c|}{ PTR } & \multicolumn{2}{|c|}{$\mathrm{EF}$} \\
\hline & Coefficient & $t$-Test & Coefficient & $t$-Test & Coefficient & $t$-Test & Coefficient & $t$-Test \\
\hline$X_{1}$ & 1.49 & N.S. & -16.44 & $<0.05$ & -0.66 & N.S. & -3.40 & N.S. \\
\hline$X_{2}$ & -0.09 & N.S. & -3.54 & N.S. & 0.08 & N.S. & -1.78 & N.S. \\
\hline$X_{1}^{2}$ & -1.63 & N.S. & 2.68 & N.S. & -1.20 & N.S. & 11.92 & N.S. \\
\hline$X_{2}^{2}$ & 0.87 & N.S. & -6.48 & N.S. & 1.30 & N.S. & -2.43 & N.S. \\
\hline$X_{1} X_{2}$ & 0.60 & N.S. & -7.45 & N.S. & 0.15 & N.S. & 3.70 & N.S. \\
\hline \multirow[t]{2}{*}{ Constant } & 90.5 & $<0.05$ & 68.2 & $<0.05$ & 91.2 & $<0.05$ & 72.7 & $<0.05$ \\
\hline & \multicolumn{2}{|c|}{$R^{2}=0.6155$} & \multicolumn{2}{|c|}{$R^{2}=0.8137$} & \multicolumn{2}{|c|}{$R^{2}=0.5413$} & \multicolumn{2}{|c|}{$R^{2}=0.4215$} \\
\hline
\end{tabular}

Table 4. Multiple Regression Analysis of Tablet Properties

\begin{tabular}{|c|c|c|c|c|c|c|c|c|}
\hline \multirow{3}{*}{ Term } & \multicolumn{4}{|c|}{$\mathrm{Mg}-\mathrm{St}$} & \multicolumn{4}{|c|}{ TR-FB } \\
\hline & \multicolumn{2}{|c|}{ TS } & \multicolumn{2}{|c|}{ DT } & \multicolumn{2}{|c|}{$\mathrm{TS}$} & \multicolumn{2}{|c|}{ DT } \\
\hline & Coefficient & $t$-Test & Coefficient & $t$-Test & Coefficient & $t$-Test & Coefficient & $t$-Test \\
\hline$X_{1}$ & -0.37 & $<0.05$ & 249 & $<0.05$ & 0.056 & N.S. & -23 & $<0.05$ \\
\hline$X_{2}$ & -0.19 & N.S. & 137 & $<0.05$ & 0.017 & N.S. & 13 & $<0.05$ \\
\hline$X_{1}^{2}$ & 0.49 & $<0.05$ & 134 & $<0.05$ & -0.040 & N.S. & 4 & N.S. \\
\hline$X_{2}^{2}$ & 0.23 & N.S. & 87 & N.S. & -0.025 & N.S. & -10 & N.S. \\
\hline$X_{1} X_{2}$ & 0.15 & N.S. & 159 & $<0.05$ & -0.130 & N.S. & -10 & N.S. \\
\hline \multirow[t]{2}{*}{ Constant } & 0.81 & $<0.05$ & 265 & $<0.05$ & 1.52 & $<0.05$ & 116 & $<0.05$ \\
\hline & \multicolumn{2}{|c|}{$R^{2}=0.9012$} & \multicolumn{2}{|c|}{$R^{2}=0.9636$} & \multicolumn{2}{|c|}{$R^{2}=0.6929$} & \multicolumn{2}{|c|}{$R^{2}=0.9236$} \\
\hline
\end{tabular}


a) $\mathrm{Mg}-\mathrm{St}$

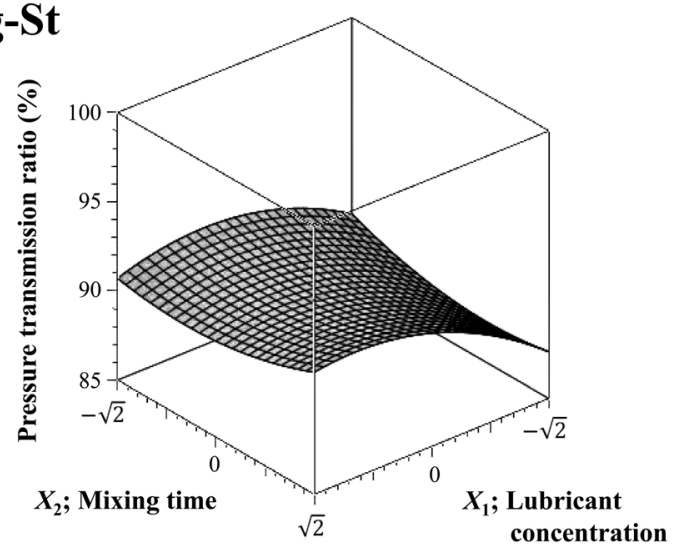

b) TR-FB

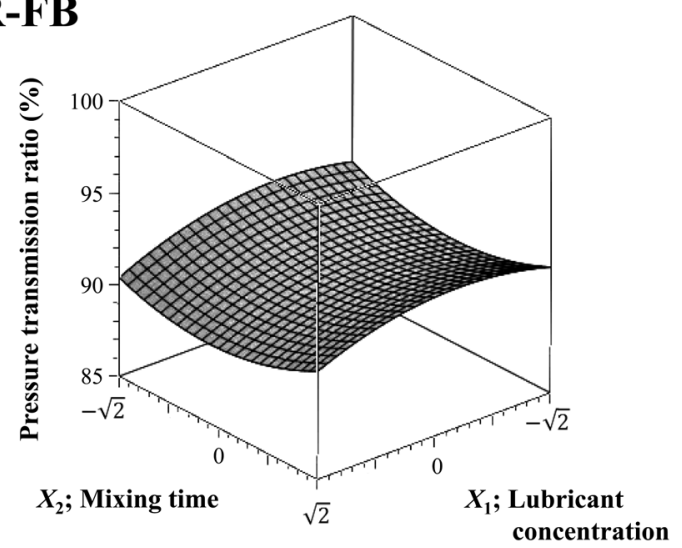

Fig. 1. Response Surface Plots of PTR as a Function of Lubricant Concentration $\left(X_{1}\right)$ and Mixing Time $\left(X_{2}\right)$

a) Mg-St, and b) TR-FB.

Pressure Transmission Ratio and Ejection Force Table 3 contains the results of the multiple regression analysis for PTR and EF, and Figs. 1 and 2 show the response surface plots for PTR and EF, respectively. Neither the lubricant concentration $\left(X_{1}\right)$ nor the mixing time $\left(X_{2}\right)$ significantly affected PTR for Mg-St or TR-FB (Table 3). This is because a high PTR value was observed even at the lowest lubricant concentration of $0.07 \%$ (Table 2). All 11 batches had EF of less than $100 \mathrm{~N}$ for both $\mathrm{Mg}-\mathrm{St}$ and TR-FB, indicating their good lubrication properties (Table 2). Figure 2 shows EF significantly decreased as $\mathrm{Mg}-\mathrm{St}$ concentration $\left(X_{1}\right)$ increased $(p<0.05)$, whereas for TR-FB, neither the lubricant concentration nor the mixing time had a significant effect. We have previously reported that when Mg-St or TR-FB was mixed with glass beads for $30 \mathrm{~min}$, a thin $\mathrm{Mg}$-St film was observed on the surface of glass beads, whereas diffusely-scattered TR-FB were observed on the surface of the glass beads even for a 30 min mixing time. ${ }^{16)}$ Therefore, the difference in EF was caused by the surface coverage of lubricants on the granules.

Tablet Tensile Strength and Disintegration Time The results of the multiple regression analysis of tablet TS and DT are shown in Table 4. For Mg-St, the terms $X_{1}$ and $X_{1}^{2}$ were significant for TS, whereas no terms were significant for TR-FB. For DT, the term $X_{1}, X_{2}, X_{1}^{2}$, and $X_{1} X_{2}$ were significant for Mg-St, and for TR-FB, the term $X_{1}$ and $X_{2}$ were significant. However, the changes in DT for TR-FB were considered to be negligible for the following reasons: DT of conventional tab- a) $\mathrm{Mg}-\mathrm{St}$

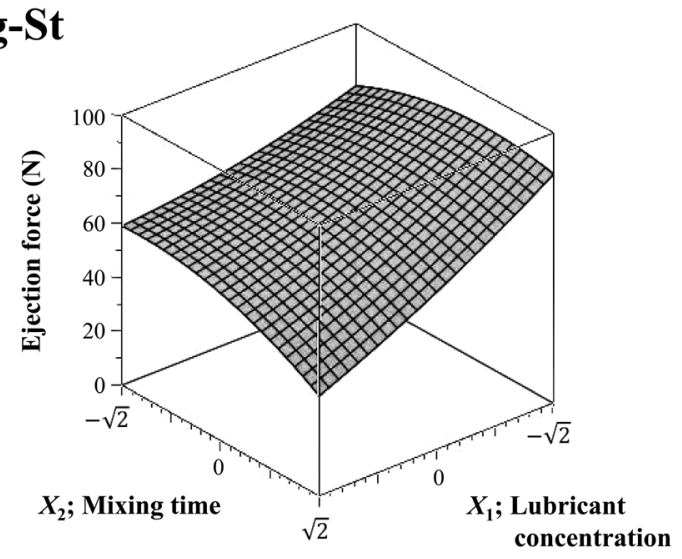

b) TR-FB

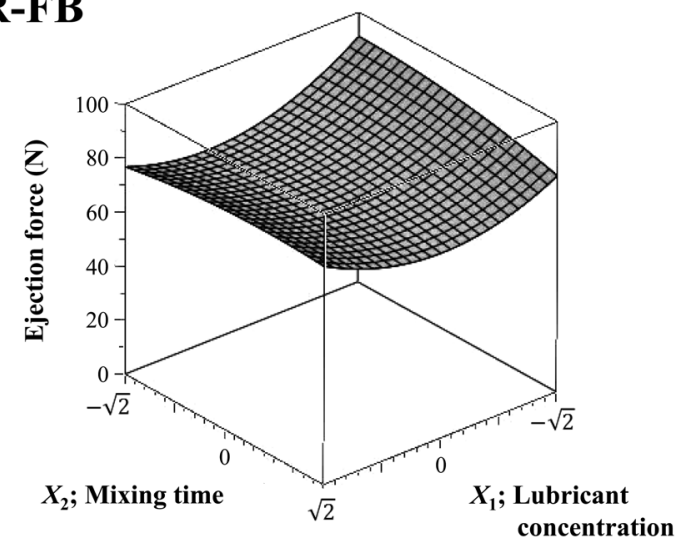

Fig. 2. Response Surface Plots of EF as a Function of Lubricant Concentration $\left(X_{1}\right)$ and Mixing Time $\left(X_{2}\right)$

a) Mg-St, and b) TR-FB.

lets is defined as less than $30 \mathrm{~min}$ in JP16; the absolute value of the coefficient for TR-FB was significantly smaller than that for Mg-St; and in this study DT for TR-FB did not change dramatically, unlike that for $\mathrm{Mg}$-St. Therefore, this indicates that even when the lubricant concentration and the mixing time were increased, respectively, TR-FB did not affect tablet TS and DT within the confidence region of the experimental design (Table 4). The response surface plots for TS and DT shown in Figs. 3 and 4, respectively, demonstrated that for Mg-St TS decreased and DT increased when the lubricant concentration and the mixing time were increased. In contrast, TS and DT for TR-FB remained unchanged. This difference between Mg-St and TR-FB was caused by delamination behavior. ${ }^{16)}$ In addition, the $X_{1} X_{2}$ term was not significant for TS, but was significant for DT in the case of Mg-St. Higher Mg-St concentrations and longer mixing times increased the surface coverage of $\mathrm{Mg}-\mathrm{St}$ on the granules. The change in DT could be explained by the increase in the thickness of the hydrophobic film on the tablet as the Mg-St concentration and the mixing time increased. In contrast, since TS depends on the amount of the surface coverage of Mg-St on the granules, TS values did not changed significantly when the granule surface was completely covered by $\mathrm{Mg}$-St, showing that the $X_{1} X_{2}$ term might not be significant for TS.

Prediction of the Useful Range of Lubricant Mixing Conditions and Experimental Validation In manufacturing processes, the lubrication and tablet properties are set to 
a) $\mathrm{Mg}-\mathrm{St}$

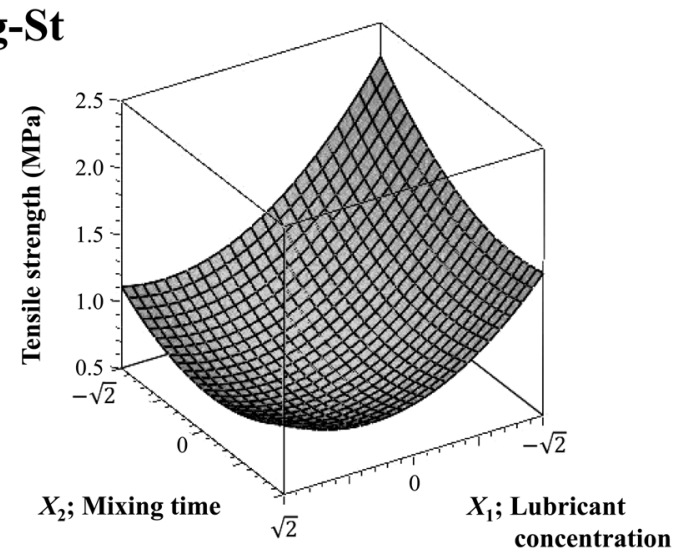

b) TR-FB

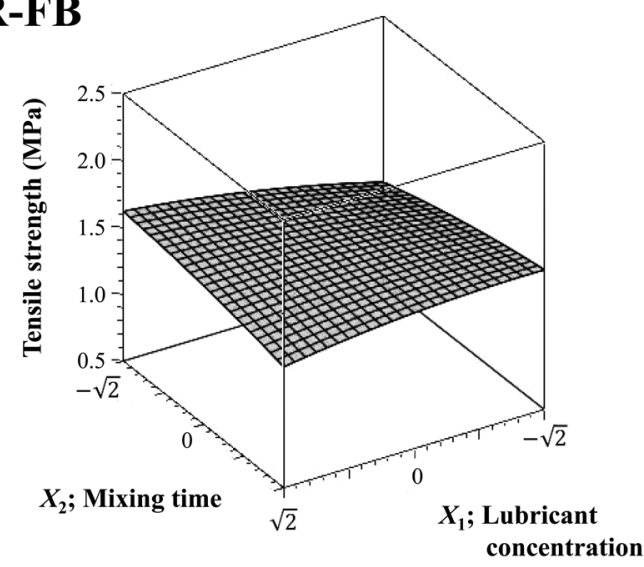

Fig. 3. Response Surface Plots of Tablet TS as a Function of Lubricant Concentration $\left(X_{1}\right)$ and Mixing Time $\left(X_{2}\right)$

a) Mg-St, and b) TR-FB.

ensure a stable supply of tablets with high quality. When PTR is greater than $90 \%$, the lubricant is considered to have good lubrication properties. We have previously reported that no tableting problems occur below EF of $160 \mathrm{~N} .{ }^{15)}$ The hardness of tablets should be greater than $50 \mathrm{~N}^{20)}$ At this hardness, tablet TS was calculated to be $1.34 \mathrm{MPa}$, using the average tablet thickness across all 11 batches $(2.96 \mathrm{~mm})$. DT of tablets should be within $30 \mathrm{~min}$ according to JP16. In addition, prediction accuracy is found to be relatively low at the external portion of the experimental design. Thus, in order to determine the range of mixing conditions which satisfies both the lubrication and tablet properties with a higher prediction accuracy, each criterion was decided as follows:

1) PTR greater than $90 \%$.

2) EF less than $160 \mathrm{~N}$.

3) TS greater than $1.34 \mathrm{MPa}$.

4) DT less than $30 \mathrm{~min}(1800 \mathrm{~s})$.

5) Within a circle with radius of $\sqrt{2}$.

The contour map displaying the relationship between the lubricant mixing conditions and the lubrication and tablet properties is shown in Fig. 5. The shaded area represents the useful range of mixing conditions which satisfy the criteria described above. The useful range of mixing conditions for $\mathrm{Mg}-\mathrm{St}$ was a combination of a lubricant concentration of $0.6-1.3 \%$ and a mixing time of $1-6.5 \mathrm{~min}$. The useful range for TR-FB are predicted to be a combination of a lubricant concentration of $0.07-3.0 \%$ and a mixing time of $1-30 \mathrm{~min}$ within the

\section{a) $\mathrm{Mg}-\mathrm{St}$}

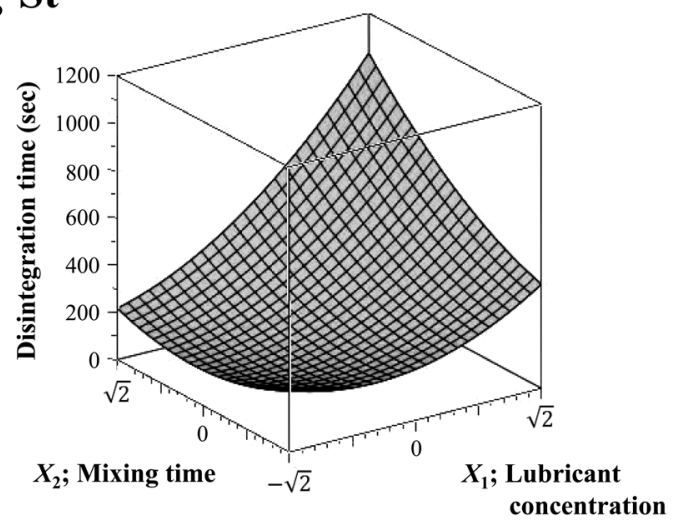

b) TR-FB

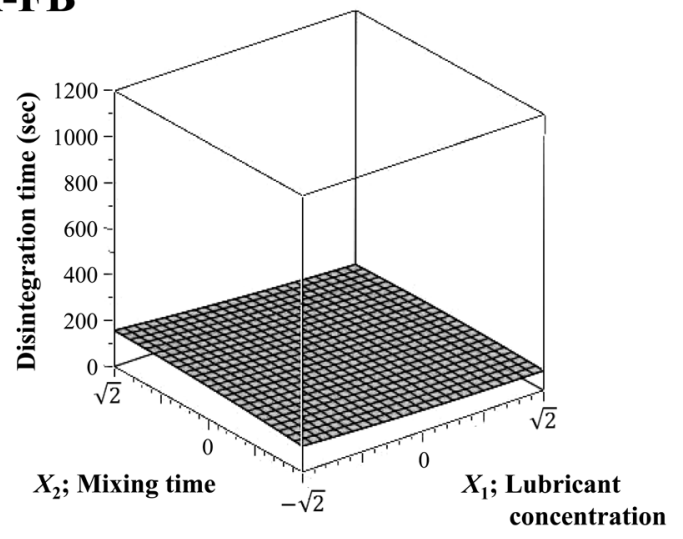

Fig. 4. Response Surface Plots of DT as a Function of Lubricant Concentration $\left(X_{1}\right)$ and Mixing Time $\left(X_{2}\right)$

a) Mg-St, and b) TR-FB.

confidence region of the experimental design, except for a concentration of $2.6-3.0 \%$ and a mixing time of $7.5-22.5 \mathrm{~min}$. This suggests that the range of mixing conditions for TR-FB is much broader than that for $\mathrm{Mg}$-St in the tablet manufacturing process; that is, the use of TR-FB enables to establish broader design space than that of $\mathrm{Mg}$-St in manufacturing process. $\mathrm{Mg}-\mathrm{St}$ is generally used at concentrations of $0.5-1.0 \%$; however, some researchers have reported that high amounts of $\mathrm{Mg}$-St up to $2.0 \%$ in the formulations was necessary to prevent tableting problem when tableting oily materials such as ibuprofenpiconol ${ }^{22)}$ and Vitamin E tocopheryl polyethylene glycol succinate, ${ }^{23)}$ suggesting that useful range of mixing conditions for $\mathrm{Mg}$-St would depend on the formulation. Although in the present study, a standard formulation given by the Society of Powder Technology, Japan, Division of Particulate Design and Preparations was used, the useful ranges for TR-FB were much broader than that for $\mathrm{Mg}$-St, indicating that TR-FB might be a potential lubricant alternative to $\mathrm{Mg}-\mathrm{St}$.

To verify the prediction accuracy of the range of mixing conditions for TR-FB, additional batches were manufactured and tested. The mixing conditions were $X_{1}$ (lubricant concentration) of $1.53 \%$ and $X_{2}$ (mixing time) of $15.5 \mathrm{~min}$. The results are given in Table 5. Each property was consistent with the predicted value and fulfilled the established criteria. Therefore TR-FB enabled the stable manufacturing of tablets satisfying the criteria within the range of mixing conditions used in this study. 
Table 5. Validation of Prediction Accuracy for Useful Range of Mixing Conditions

\begin{tabular}{|c|c|c|c|c|c|}
\hline \multirow{2}{*}{ Mixing condition } & \multirow{2}{*}{ Type } & \multicolumn{4}{|c|}{ TR-FB } \\
\hline & & PTR (\%) & $\mathrm{EF}(\mathrm{N})$ & $\mathrm{TS}(\mathrm{MPa})$ & DT (s) \\
\hline$X_{1} ; 1.53 \%$ & Predicted & 91.2 & 72.6 & 1.52 & 116 \\
\hline \multirow{2}{*}{$X_{2} ; 15.5 \mathrm{~min}$} & Experimental & 93.4 & 91.0 & 1.95 & 109 \\
\hline & Criterion & $>90.0$ & $<160$ & $>1.34$ & $<1800$ \\
\hline
\end{tabular}

\section{a) $\mathrm{Mg}-\mathrm{St}$}

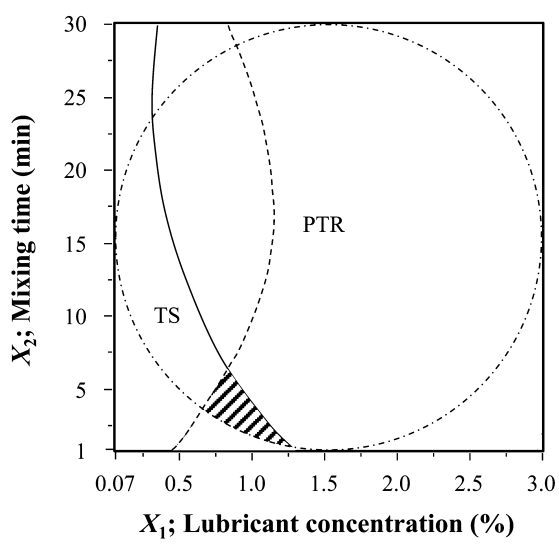

b) TR-FB

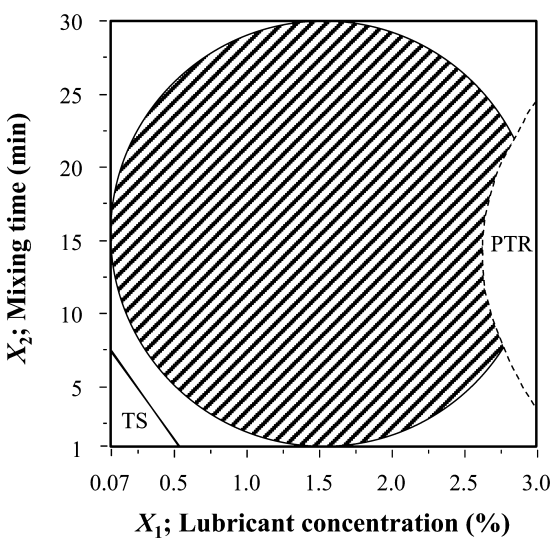

Fig. 5. Contour Map Showing the Relationship between the Lubricant Mixing Conditions and the Lubrication and Tablet Properties

a) Mg-St, and b) TR-FB. Shaded area; useful range of mixing conditions which satisfy lubrication and tablet properties, dashed line; criterion of PTR, solid line; criterion of TS, dashed-dotted line; boundary line of prediction accuracy associated with the experimental design.

\section{Conclusion}

$\mathrm{Mg}-\mathrm{St}$ is a widely used lubricant in tablet manufacturing. However, changes in the mixing conditions of $\mathrm{Mg}-\mathrm{St}$ often influence quality attributes, such as tablet TS and DT. Aiming to solve this problem, we used experimental design and multiple regression analysis and demonstrated for the first time that the useful range of mixing conditions is broader for TR-FB than for $\mathrm{Mg}-\mathrm{St}$. At a lubricant concentration of $0.07-3.0 \%$ and mixing time of 1-30 min, TR-FB showed good lubrication properties. Changes in the mixing conditions of TR-FB did not affect the tablet properties, such as TS and DT. This suggests that TR-FB allows tablets with high quality attributes to be produced consistently. In addition, it was demonstrated that
TR-FB was effective over the broad range of standard mixing conditions: concentrations of $0.07-3.0 \%$ and mixing times of 1-30 min, with the standard formulation given by the Society of Powder Technology, Japan/Division of Particulate Design and Preparations. This implies that the use of TR-FB enables to establish broader design space than that of $\mathrm{Mg}-\mathrm{St}$ in manufacturing process and that TR-FB might be suitable for other different type of granules or formulations. Therefore, TR-FB is an attractive alternative lubricant to $\mathrm{Mg}-\mathrm{St}$.

Acknowledgments The authors thank Riken Vitamin Co., Ltd., DFE Pharma, Nihon Shokuhin Kakou Co., Ltd., and Nippon Soda Co., Ltd. for providing the excipients used in this study.

\section{References}

1) Strickland W. A. Jr., Nelson E., Busse L. W., Higuchi T., J. Am. Pharm. Assoc. Am. Pharm. Assoc., 45, 51-55 (1956).

2) Udeala O. K., Onyechi J. O., Agu S. I., J. Pharm. Pharmacol., 32, 6-9 (1980).

3) Flores L. E., Arellano R. L., Esquivel J. J. D., Drug Dev. Ind. Pharm., 26, 297-305 (2000).

4) Uğurlu T., Turkoglu M., Int. J. Pharm., 353, 45-51 (2008).

5) Levy G., Gumtow R. H., J. Pharm. Sci., 52, 1139-1144 (1963).

6) Murthy K. S., Samyn J. C., J. Pharm. Sci., 66, 1215-1219 (1977).

7) Rajala R., Laine E., Thermochim. Acta, 248, 177-188 (1995).

8) Wada Y., Matsubara T., Powder Technol., 78, 109-114 (1994).

9) Leinonen U. I., Jalonen H. U., Vihervaara P. A., Laine E. S., J. Pharm. Sci., 81, 1194-1198 (1992).

10) Barra J., Somma R., Drug Dev. Ind. Pharm., 22, 1105-1120 (1996).

11) Hölzer A. W., Sjögren J., Int. J. Pharm., 2, 145-153 (1979).

12) Chowhan Z. T., Chi L. H., J. Pharm. Sci., 75, 542-545 (1986).

13) Salpekar A. M., Augsburger L. L., J. Pharm. Sci., 63, 289-293 (1974).

14) Aoshima H., Miyagishima A., Nozawa Y., Sadzuka Y., Sonobe T., Int. J. Pharm., 293, 25-34 (2005).

15) Uchimoto T., Iwao Y., Ikegami Y., Murata T., Sonobe T., Miyagishima A., Itai S., Int. J. Pharm., 386, 91-98 (2010).

16) Uchimoto T., Iwao Y., Takahashi K., Tanaka S., Agata Y., Iwamura T., Miyagishima A., Itai S., Eur. J. Pharm. Biopharm., 78, 492-498 (2011).

17) Food and Drug Administration, "Final report on pharmaceutical cGMPs for the 21st century-A risk-based approach," 2003.

18) Kimura S., Iwao Y., Ishida M., Uchimoto T., Miyagishima A., Sonobe T., Itai S., Int. J. Pharm., 391, 244-247 (2010).

19) Otsuka T., Iwao Y., Miyagishima A., Itai S., Int. J. Pharm., 409, 81-88 (2011)

20) Sano S., Iwao Y., Kimura S., Itai S., Int. J. Pharm., 416, 252-259 (2011).

21) Fell J. T., Newton J. M., J. Pharm. Sci., 59, 688-691 (1970).

22) Watanabe Y., Takei M., Koizumi K., Yamada N., Utoguchi N., Matsumoto M., Drug Delivery System, 12, 409-413 (1997).

23) Jin F., Tatavarti A., Int. J. Pharm., 389, 58-65 (2010). 